\title{
Determining the Role of Helicobacter pylori in Chronic Sinus Infections Using the Polymerase Chain Reaction
}

\author{
Soheila Nikakhlagh ${ }^{1}$; Ali Reza Samarbafzadeh ${ }^{1}$; Mojtaba Jahani ${ }^{1}$; Hamed Poostchi ${ }^{1}$;Gholam \\ Abbas Kayedani ${ }^{1}$; Maryam Naghashpoor ${ }^{1}$; Nader Saki ${ }^{2, *}$ \\ ${ }^{1}$ Hearing \& speach Research Centre, Ahvaz Jundishapur University of Medical Sciences, Ahvaz, IR Iran \\ ${ }^{2}$ Health Research Institute, Infectious and Tropical Diseases Research Center, Ahvaz Jundishapur University of Medical Sciences, Ahvaz, Iran \\ ${ }^{*}$ Corresponding author: Nader Saki, Health Research Institute, Infectious and Tropical Diseases Research Center, Ahvaz Jundishapur University of Medical Sciences, Ahvaz, Iran. Fax: \\ +98-6132921838, E-mail: Saki-n@ajums.ac.ir
}

Received: May 29, 2014; Revised: September 17, 2014; Accepted: October 31, 2014

\begin{abstract}
Background: Helicobacter pylori are becoming increasingly recognized as a possible pathological cause of chronic rhinosinusitis (CRS). Objectives: Considering the prevalence of CRS and its impact on quality of life, we decided to determine the role of $H$. pylori in chronic sinus infections by using the PCR technique.

Patients and Methods: In a case-control analytical epidemiologic survey, the study population was selected by consecutive sampling from patients with CRS undergoing endoscopic sinus surgery during years 2010 - 2012. Patients were divided into two groups. The study group consisted of patients with CRS and the control group consisted of patients with nasal obstruction caused by concha bullosa, without inflammation or infection of the sinuses. Sampling was performed during surgery from the infected tissue and from the middle turbinate mucosa. Eventually, bacterial DNA was extracted and used for the PCR test, in order to isolate H.pylori.

Results: Nine patients (18\%) with CRS had H. pylori isolated from their samples whereas in the control group, H. pylori were only found in two patients $(4 \%)$; this difference was statistically significant $(\mathrm{P}=0.025)$. The indicator wasn't statistically significant between males and females. There was no statistical correlation in relative frequency of $H$. pylori for different age groups $(\mathrm{P}>0.05)$.

Conclusions: There was a significant correlation between CRS and presence of $H$. pylori in sinonasal mucosa. This relationship may reflect the role of $H$. pylori as one of the pathogenic factors in the development of CRS. However, further studies are required to confirm this role.
\end{abstract}

Keywords: Infection; Helicobacter pylori; Polymerase Chain Reaction

\section{Background}

Chronic rhinosinusitis (CRS) is a disease that significantly affects the patient's quality of life. Regarding the pathophysiology of the disease, several etiologies have been proposed including obstructive factors, fungi, biofilm, and bacterial agents with different mechanisms such as Staphylococcus aureus as a super antigen and Helicobacter pylori as a possible pathological cause for chronic rhinosinusitis (1-5).

Studies indicate the high prevalence of gastroesophageal reflux in patients with chronic rhinosinusitis. Experimental studies indicate that after exposure of nasopharyngeal mucosa to gastric acid, eustachian tube dysfunction occurs. Reflux of stomach content into the nasopharynx and nose causes edema in nasopharynx and sinonasal mucosa, which will lead to disruption in pressure regulation and clearing of the middle ear and paranasal sinuses. This process prepares the way for inflammation in diseases of the upper respiratory system (6-8). Helicobacter pylori are Gram negative and microaerophilic microorganisms. Infections caused by H. pylori are common bacterial infections in humans with a global spread. Prevalence of the disease in developed countries is $30-40 \%$ while it is $80-90 \%$ in developing countries. Human gastric system was thought to be the sole source of $H$. pylori until it was found in human dental plaques, mouth, saliva, tonsils, adenoid tissue, middle ear, nasal polyps and sinus mucosa $(1,7,8)$. Today, $H$. pylori is not only recognized as a cause of gastritis, but it is also known as a microorganism causing systemic infections $(6,8,9)$. Studies suggest that H.pylori infected gastric fluid enters the nasopharyngeal cavity by gastroesophageal reflux disease (GERD) and colonizes in dental plaques, adenoid tissues and tonsils $(7,8,10)$. The bacteria ascend to the middle ear and paranasal sinuses directly or by reflux from these locations and may trigger some pathological changes. However, the exact mechanism of action remains unclear. Isolation of $H$. pylori from the respiratory and gastrointestinal tracts in several studies suggests that there may be a close relationship between $H$. pylori and pathogenesis of various ear, nose and throat problems. Detection of $H$. py-

Copyright (C) 2015, Ahvaz Jundishapur University of Medical Sciences. This is an open-access article distributed under the terms of the Creative Commons Attribution-NonCommercial 4.0 International License(http://creativecommons.org/licenses/by-nc/4.0/) which permits copy and redistribute the material just in noncommercial usages, provided the original work is properly cited. 
lori in nasal mucosa of patients with CRS has significantly increased by the PCR method $(5,6,8)$.

\section{Objectives}

Considering that rhinosinusitis is a common public health issue and treatment of CRS is not easy, determining the pathogenesis and pathological mechanisms of this disease is extremely important. With regards to the prevalence of CRS and its impact on patients' quality of life, the aim of this study was to detect $H$. pylori in the middle turbinate mucosa of patients with CRS, and determine the association of $H$. pylori with CRS.

\section{Patients and Methods}

\subsection{Study Population and Design}

In this case-control study, the subjects were consecutively selected from patients who had referred to educational hospitals and had a diagnosis of chronic rhinosinusitis or nasal obstruction caused by concha bullosa (50 patients with chronic rhinosinusitis and 50 patients with concha bullosa), during August 2010 to June 2012. All patients underwent functional endoscopic sinus surgery (FESS). Patients were divided to two groups: the study group included patients with refractory CRS and the control group included patients with concha bullosa without inflammation and sinus infection.

We included subjects with rhinosinusitis symptoms for over 12 weeks and proof of anterior and posterior ethmoid sinuses inflammation by imaging (CT scan) of at least one side, with or without involvement of sphenoid sinus, after at least four weeks of antibiotic therapy. In the control group, we included those with complaints of nasal congestion and proof of existence of obstruction causing middle turbinate pneumatization by imaging (CT scan). We excluded those with a history of antacid use, use of H2-blocker and proton pump inhibitor drugs a week before surgery, cystic fibrosis, immotile-cilia syndrome and immune deficiency, from both groups; furthermore, symptoms of fungal sinusitis in CT scan in the study group and involvement and opacification of sinuses in the control group were other exclusion criteria. Factors included age, gender and $H$. Pylori status.

\subsection{DNA Extraction and Polymerase Chain Reac- tion}

The samples were taken during surgery from the infected tissue in the case group and from the middle turbinate mucosa in the control group. Next, bacterial DNA was extracted by the ROCHE extraction kit and the extracted DNA was used as a template for PCR. The primer pairs used for PCR included BabA2s SA, SB, Cag1, Cag2, Cag3 and Cag4, which are appropriate primers for amplification of all $H$. pylori strains. The PCR products were elec- trophoresed on agarose gel (2\%) and stained by ethidium bromide. The intended band (bp520) was observed in all positive samples.

\subsection{Statistical Analysis}

Data analysis was performed for the 100 subjects including 50 patients in the case group and 50 subjects in the control group. Data analysis was performed using the SPSS (version 18.0) software (Statistical Procedures for Social Sciences; Chicago, Illinois, USA). Chi-square, Fisher, and independent sample's t-test were used and were considered statistically significant at P values less than 0.05 .

\section{Results}

In the study group, 50 patients with chronic rhinosinusitis including 45 (90\%) males and five (10\%) females underwent sinus endoscopy surgery. In the control group, there were 50 patients with concha bullosa including 26 (52\%) males and 24 (48\%) females who underwent sinus endoscopy surgery. There was no difference in frequency distribution of genders across the two groups $(P>0.05)$. The patients with CRS were in the age range of $22-74$ years, with a mean age of $39.6 \pm 8.7$ years. In the control group, the age range of patients was 7 - 60 years, with a mean age of $29.7 \pm 7.2$ years. In this study, sinus samples of nine patients (18\%) from 50 patients with chronic rhinosinusitis were positive for H. pylori, (Chart 1) while in the control group, H. pylori was found only in two patients (4\%) from patients with concha bullosa; this difference was statistically significant $(\mathrm{P}=0.025)$.

Of the nine patients in the CRS group, eight patients $(17.8 \%)$ were male and one patient $(20 \%)$ was female. Of the two patients in the control group, one patient (3.8\%) was male and one patient (4.2\%) was female. According to these results, the difference between the two groups was not statistically significant in terms of the relative frequency of $H$. pylori in male patients $(\mathrm{P}>0.05)$. Moreover, this indicator in females was not statistically significant between the two groups $(\mathrm{P}>0.05)$. There was no statistically significant difference between the case and control groups in terms of relative frequency of $H$. pylori in different age groups ( $\mathrm{P}$ $>0.05$ ). Odds ratio (OR) of incidence of $H$. pylori in patients with CRS and $H$. pylori in patients with concha bullosa was determined to be 5.26. In this study, the sensitivity of H. pylori was $81.8 \%$ and the specificity was $96 \%$.

Figure 1. Polymerase Chain Reaction Positivity for Helicobacter pylori 


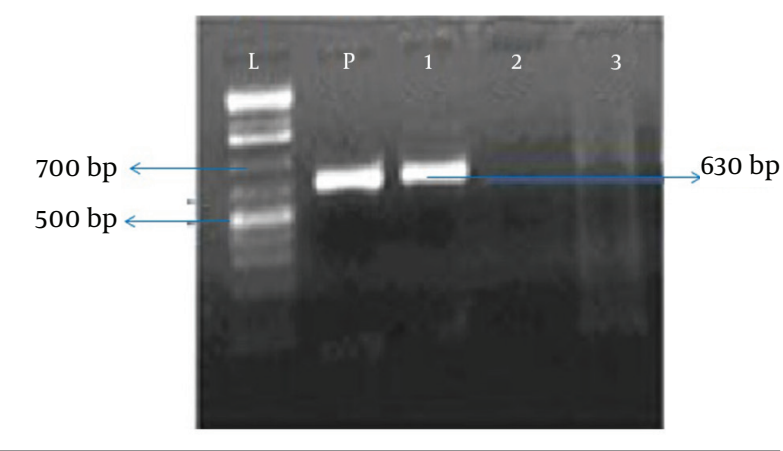

$\mathrm{L}=$ Ladder (100 bp), $\mathrm{P}=$ Positive control, lanes are numbered in the Following order from 1 to 2 : case H.pylori-positive (286 bp), case H. pylori-negative

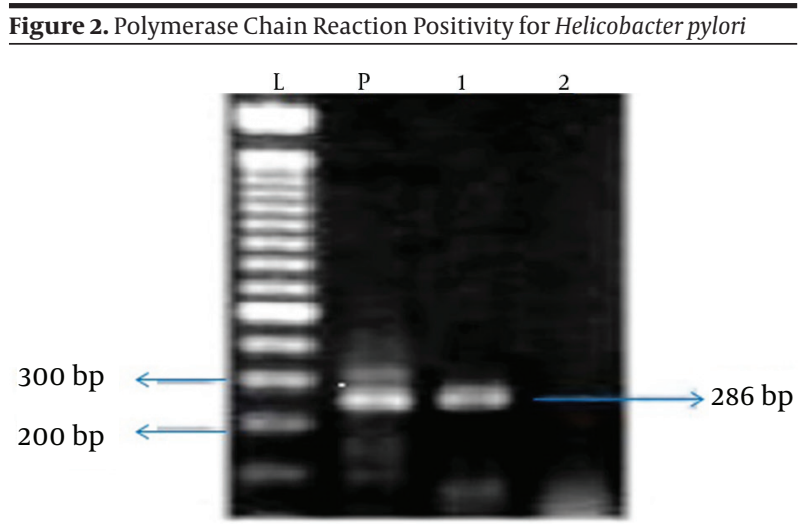

$\mathrm{L}=$ Ladder (100 bp), $\mathrm{P}=$ Positive control, lanes are numbered in the following order from 1 to 3 : case H. pylori-positive (630 bp), negative control, case H. pylori-negative

\section{Discussion}

Chronic rhinosinusitis is one of the most common chronic diseases which significantly reduces quality of life, in addition to the costs imposed on the health system (4). There has been increasing evidence regarding $H$. pylori and reflux as possible pathological causes for chronic rhinosinusitis. Studies have indicated high prevalence of GERD in patients with chronic rhinosinusitis. In addition drug therapy of GERD together with antibiotics has improved symptoms of sinusitis in $79 \%$ of patients (11). Isolation of $H$. pylori from the respiratory and gastrointestinal tracts in several studies suggests that there may be a close relationship between $H$. pylori and pathogenesis of various ear, nose and throat problems (12). Detection of $H$. pylori in nasal mucosa of patients with CRS has significantly increased by the PCR method. In this study, the PCR method was used to identify H.pylori in sinonasal mucosa samples. The method used in some of the studies conducted so far was PCR and in some others, methods such as the urease test (CLO test) or immunohistological analysis were used $(13,14)$. In previous studies, the sample size of the study and control groups was smaller than the current study.
This study was conducted with a larger number of patients in CRS and concha bullosa groups than the previous studies, in order to better represent the possible relationship between $H$. pylori and CRS. Helicobacter pylori were found in sinonasal mucosa of $18 \%$ of patients with CRS while they were observed in only $4 \%$ of sinonasal samples of patients with concha bullosa where there was a statistically significant difference between the two groups. In a number of studies in which immunohistological and Campylobacter-like organism test (CLO) test methods were used, the relative frequency of $H$. pylori in sinonasal samples of the study group was reported between $20-26 \%$ and between $0-3.4 \%$ in the control group, where there were statistically significant differences between the two study and control groups. However, another study conducted with these two methods, reported no statistically significant difference between the two groups in terms of relative frequency of $H$. pylori (15).

In a study conducted by Ozdek et al. using the PCR method to detect $H$. pylori, the relative frequency of $H$. pylori in patients with chronic rhinosinusitis was $33 \%$, whereas none of the controls were positive for H. pylori (16). However, in another study, which also used the PCR method, the relative frequency of $H$. pylori in sinonasal samples of the study and control groups was similar with no statistically significant difference (14). Some studies have also been conducted in Iran with different results. For instance, Noorbakhsh et al. in 2007 conducted a study on the patients' polyp tissue and inferior turbinate of control group and compared the serum levels of antibodies against $H$. pylori and its DNA in tissues, and found that $H$. pylori was positive in $32.3 \%$ of patients and $4 \%$ of controls; furthermore the authors suggested that PCR is the best method for detection of $H$. pylori infections (17). However, in a study conducted by Khademi et al. during years 2006 to 2008 , PCR was negative in all patients with nasal polyps and in the control group (18). Based on the results of this study it can be concluded that there is a significant relationship between CRS and presence of $H$. pylori in sinonasal mucosa. This relationship may reflect the role of H. pylori as one of the pathogenic factors in the development of chronic rhinosinusitis. However, further studies with larger sample sizes are required to confirm the role of H. pylori in CRS.

\section{Acknowledgements}

The authors would like to thank the research deputy of Ahavz Jundishapur University of Medical Sciences for their valuable cooperation. This research was part of a General physician thesis (Ref-No.89191).

\section{Authors' Contributions}

All authors had equal role in design, work, statistical analysis and manuscript writing. 


\section{Funding/Support}

Ahavz Jundishapur University of Medical Sciences provided funding for this research.

\section{References}

1. Saki N, Nikakhlagh S, Ahmadi K. Comparison of PCR assay and culture for detecting bacteria in middle ear fluid of children with otitis media with effusion. Int Adv Otol. 2009;5(1):31.

2. Nikakhlagh S, Saki N, Baghbdrani RA, Rahim F, Sheikh AFZ. Microbiology of Adenoid Infection in Children with Recurrent of Otitis Media. Asian J Bio Sci. 2011;4(3):252-8.

3. Saki N, Ardeshir Larijani F, Rahim F. The association of Helicobacter Pylori infection with otitis media: a meta-analysis. Arch Des Sci. 2013;66:452-70.

4. Rudmik L, Smith TL. Quality of life in patients with chronic rhinosinusitis. Curr Allergy Asthma Rep. 2011;11(3):247-52.

5. Kim HY, Dhong HJ, Chung SK, Chung KW, Chung Y], Jang KT. Intranasal Helicobacter pylori colonization does not correlate with the severity of chronic rhinosinusitis. Otolaryngol Head Neck Surg. 2007;136(3):390-5.

6. Kurtaran H, Uyar ME, Kasapoglu B, Turkay C, Yilmaz T, Akcay A, et al. Role of Helicobacter pylori in pathogenesis of upper respiratory system diseases. J Natl Med Assoc. 2008;100(10):1224-30.

7. Gasbarrini A, Franceschi F, Gasbarrini G, Pola P. Extraintestinal pathology associated with Helicobacter infection. Eur J Gastroenterol Hepatol.1997;9(3):231-3.

8. Morinaka S, Ichimiya M, Nakamura H. Detection of Helicobacter pylori in nasal and maxillary sinus specimens from patients with chronic sinusitis. Laryngoscope. 2003;113(9):1557-63.
9. Ozmen S, Yucel OT, Sinici I, Ozmen OA, Suslu AE, Ogretmenoglu O, et al. Nasal pepsin assay and $\mathrm{pH}$ monitoring in chronic rhinosinusitis. Laryngoscope. 2008;118(5):890-4.

10. Ozcan C, Polat A, Otag F, Gorur K. Does Helicobacter pylori play a role in etiology of nasal polyposis? Auris Nasus Larynx. 2009;36(4):427-30.

11. Dinis PB, Subtil J. Helicobacter pylori and laryngopharyngeal reflux in chronic rhinosinusitis. Otolaryngol Head Neck Surg. 2006;134(1):67-72.

12. Cvorovic L, Brajovic D, Strbac M, Milutinovic Z, Cvorovic V. Detection of Helicobacter pylori in nasal polyps: preliminary report. J Otolaryngol Head Neck Surg. 2008;37(2):192-5.

13. Szczygielski K, Jurkiewicz D, Rapiejko P. [Detection of Helicobacter pylori in nasal polyps specimens using urease test GUT plus]. Pol Merkur Lekarski. 2005;19(111):309-11.

14. Ozyurt M, Gungor A, Ergunay K, Cekin E, Erkul E, Haznedaroglu T. Real-time PCR detection of Helicobacter pylori and virulenceassociated cagA in nasal polyps and laryngeal disorders. Otolaryngol Head Neck Surg. 2009;141(1):131-5.

15. Koc C, Arikan OK, Atasoy P, Aksoy A. Prevalence of Helicobacter pylori in patients with nasal polyps: a preliminary report. Laryngoscope. 2004;114(11):1941-4.

16. Ozdek A, Cirak MY, Samim E, Bayiz U, Safak MA, Turet S. A possible role of Helicobacter pylori in chronic rhinosinusitis: a preliminary report. Laryngoscope. 2003;113(4):679-82.

17. Noorbakhsh S, Tabatabaei A, Farhadi M, Shamshiri A. Helicobacter pylori-DNA in nasal polyp tissues in compare with inferior nasal turbinate tissues: A case-control study in Tehran, Iran. J Infect Dis Immun. 2011;3(1):37-8.

18. Khademi B, Chohedri A, Azar Pira N, Ashraf MJ. Helicobacter Pylori in nasal Polyposis. Professional Med J. 2012;19:455-61. 